\title{
Joint power and rate allocation in CDMA-based underlay cognitive radio networks for a mixture of streaming and elastic traffic
}

\author{
Mina Dashti ${ }^{\text {* }}$ and Paeiz Azmi ${ }^{2}$
}

\begin{abstract}
This article investigates the issue of radio resource allocation strategies for cognitive networks based on the underlay approach, while adhering to the interference constraint on the primary user. Joint rate and power allocation problem is considered for secondary users (SUs) with homogeneous and heterogenous traffic subject to the QoS and interference threshold constraints. Two well-known fairness approaches [max-min and proportional fairness (PF)] are compared for the proposed optimization problem. Three scenarios are considered. The first scenario corresponds to elastic traffic in which all the SUs are elastic users. In the second, it is assumed that all the SUs are streaming users. Considering the proportional and max min fairness, it is observed that for both fairness criterion the streaming users achieving higher throughput and fairness, compared with the elastic users due to the fact that for streaming users, stringent transmission rate guarantees are necessary to ensure real-time communication. Moreover, considering the requirements of future wireless networks, a cross-layer resource-allocation is proposed for heterogeneous traffic in the third scenario. A combination of streaming traffic (which requires a maximum guaranteed average delay) and elastic traffic (with flexible rate requirements) is investigated. The optimization problem allocates the available resources to the streaming users such that the delay constraints of the streaming users are satisfied. Through extensive simulations, the effect of streaming traffic, Interference threshold, minimum processing gain, imperfect channel state information and signal-to-interference-noise ratio constraint on the total throughput of elastic users are investigated. Simulation results demonstrate that in all scenarios PF outperforms max min fairness. Furthermore, it is shown that increasing the number of streaming traffic results in lower throughput of elastic users for both PF and max min fairness.
\end{abstract}

\section{Introduction}

Cognitive radio which enables spectrum sharing between different wireless services attempts to overcome spectrum scarcity [1-4]. In cognitive radio networks, unlicensed user or secondary user ( $\mathrm{SU})$ is allowed to share spectrum with licensed user, known as primary user (PU). As defined clearly in [5], there are three basic techniques for spectrum sharing: overlay, interweaving, and underlay. In the overlay scheme, the SU detects the presence of the PUs, and changes its own transmitted signal so that it does not imposed harmful interference on the communication of the PUs. In the interweaving approach, the secondary detect and exploit portions of the spectrum that are not

\footnotetext{
*Correspondence: dashtimina@ieee.org

1 Department of Electrical Engineering, Science and Research Branch, Islamic Azad University, Tehran, Iran

Full list of author information is available at the end of the article
}

being occupied by licensed services are referred to as spectrum holes in order to avoid interference with the PUs. In the case of underlay principle, the $\mathrm{SU}$ is allowed to use the spectrum of the PUs only when the interference received from the $\mathrm{SU}$ is less than the interference level that is tolerable by the primary receiver.

spectrum sharing in cognitive radio has recently attracted growing attention in the literature. The opportunistic spectrum sharing approach in the code-division multiple access (CDMA)-based uplink MAC over the cognitive radio networks was proposed in [6] to achieve the tradeoff between the interference imposed into the PUs and overall spectrum utilization. Problems of channel sensing, data transmission, and power and rate allocations were considered.

The problem of simultaneous power and code-channel allocation for secondary transmission links co-existing 
with an unknown primary CDMA system was considered in [7]. An iterative least square based primary identification algorithm which could blindly detect the code channels utilized by PUs was developed. It was shown that this method did not require any prior knowledge about the primary networks.

The authors of [8] presented a soft decision spectrally modulated, spectrally encoded framework to achieve synergistic CR benefits of overlay and underlay schemes. Analytic derivation of $\mathrm{CR}$ error probability for overlay and underlay applications for a family of SMSE signals, including OFDM and MC-CDMA was provided. It was shown that the underlay cognitive radio waveform was able to adapt its bandwidth based on user requirements and environmental conditions. Simulated performance analysis demonstrated that the hybrid overlay/underlay waveform could be utilized to improve spectrum efficiency.

An algorithm for calculating the adaptive transmission parameters in a cognitive DS MC-CDMA system was proposed in [9] and it was shown that this algorithm combated interference in the channel effectively.

This article specifically focuses on the underlay spectrum sharing approach. This approach has attracted significant attention (see, e.g., [10-13]). In wireless communications, radio resource allocation (RRA) is vital in controlling how scarce resources can be allocated. The studies related to RRA for SUs in the underlay spectrum sharing scheme are relevant to our work.

The authors of [14] focused on the proportional fairness (PF) in a CDMA-based cognitive wireless network with spectrum underlay where the PUs could increase transmit power to counterbalance the harmful interference caused by the SUs. The authors of [15] presented a two-phase channel and power allocation approach for CDMA-based underlay cognitive radio networks to improve the system throughput. The authors of [12] focused on the CDMAbased underlay cognitive radio systems where channelaware access control algorithm was proposed to protect the primary transmission. In this article, the access probabilities of SUs were adjusted based on the channel-state information and the measured interference temperature.

In [16], the problem of joint rate and power allocation was formulated by considering QoS constrains [in terms of minimum required signal-to-interference-noise ratio (SINR)] for different unlicensed users and also interference temperature constraints for the PUs. The main aim was to maximize the total transmission rate of the SUs by adjusting the transmit power of these users. Resource allocation for spectrum underlay in CDMA networks under QoS and interference constraints were presented in [17]. Joint power and rate allocation with max-min fairness and PF were considered. Coexistence of cognitive radio and CDMA networks were studied in [18], where a more flexible scheme that utilized the average channel gain was considered. In [16-18], resource allocation in underlay cognitive radio was conducted in each time slot of packet transmissions. In [19], PF was achieved over multiple time slots.

In this article, we develop the joint rate and power allocation problem subject to Qos and interference threshold constraints. We also provide comparison analysis between two popular fairness concepts: $\max -\min$ and PF. In maxmin fairness, the minimum achievable data rate is maximized. Max-min fairness ignores revenue maximization for the network operators; but, rather the focus is on the satisfaction of service fairness from the users perspective. $\mathrm{PF}$ is defined in terms of maximizing total logarithmic user throughput. The PF improves the trade off between the total network performance in terms of throughput and fairness experience of individual users.

We first consider homogeneous traffic for two cases; one in which we consider that all the SUs are elastic users with flexible rate requirements and one in which we assume that all the SUs are streaming users which correspond to the real-time transfer of various signals (e.g., voice, streaming audio/video) that requires a maximum guaranteed average delay. Simulation results show that streaming traffic achieve higher throughput than of the elastic traffic for both PF and max-min fairness. This results from the fact that, for streaming applications, stringent transmission rate guarantees are necessary to ensure real-time communication. Our results also indicate that resource allocation solutions are fairer for the streaming traffic in comparison to elastic users.

In [16-19], resource allocation in underlay spectrum sharing is only conducted for homogeneous traffic. However, next-generation wireless networks are anticipated to support multimedia traffic. With heterogeneous traffic, quality of service provisioning and fairness support are also imperative.

In this article, we further extend the related literature by incorporating a mixture of different traffic types. It is in line with the fact that in the next wireless networks, diversified services are provided for different traffic types with various delay requirements. The formulation is then extended to the case of heterogeneous traffic. The considered combination includes streaming traffic and elastic traffic. The main aim is to maximize the total throughput of elastic users where the average delay constraint for streaming traffic and the maximum transmission power, rate, and interference constraint are satisfied. Using simulations, we evaluate the impact of streaming traffic on the total throughput of elastic users. Simulation results are provided, which show the increase in throughput of elastic users achieved by decreasing number of streaming users. A discussion of the effects of interference threshold, minimum process gain, and SINR constraint on the throughput of SUs are also performed. 
The remainder of the article is organized as follows. Section "System model" provides the system model and main assumptions. The joint power and rate assignment problem formulation for different objective functions based on a fair and efficient framework for homogeneous traffic is discussed in Sections "RRA for elastic traffic" and "RRA for streaming traffic". Furthermore, RRA based on heterogeneous delay requirements are investigated in Section "RRA for heterogeneous traffic". Finally, simulation results are demonstrated in Section "Simulation results", followed by concluding remarks in Section "Conclusion".

\section{System model}

We consider a CDMA-based cellular network as the primary network, in which mobile stations (MSs) or PUs communicate with the corresponding base station through uplink transmission. Spectrum utilization may be very low for some periods of time, and in some specific locations; therefore, some MSs may form a secondary ad hoc network and communicate directly with each other. Figure 1 shows the system model considered in this article.

Allowing ad hoc communications within a cellular coverage has been studied in the related literature (see, e.g., [19]). Secondary transmissions use the same frequency

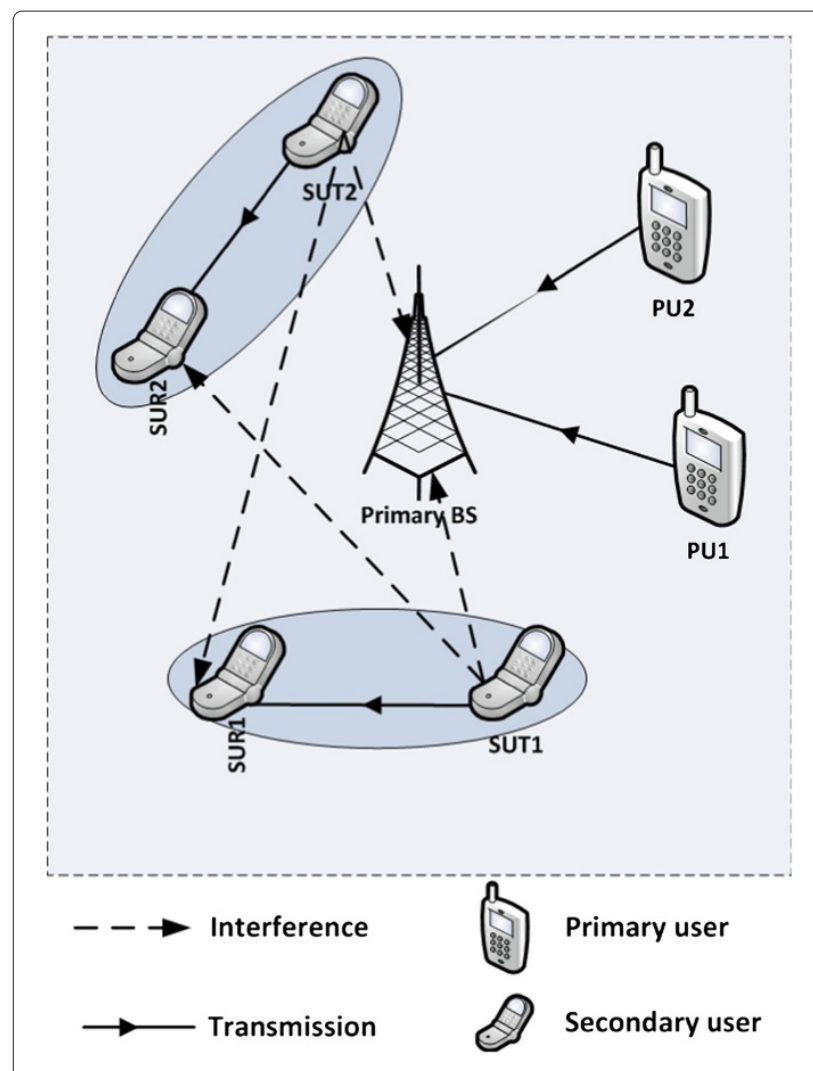

Figure 1 System model. The considered system model in this article. band as the uplink in the primary network and interfere with the uplink transmissions in the primary network. $I_{\mathrm{t} h}$ is utilized to denote the maximum interference limit tolerable at the primary receiving point (i.e., base station).

Rate and power allocations for SUs are performed for both homogeneous and heterogeneous traffics. Transmitter $i$ and receiver $i$ are used to indicate secondary receiver of link $i$ and $N_{s}$ is the total number of SUs. The transmission rate and the transmission power corresponding to the $i$ th secondary link are $R_{i}$ and $P_{i}$, respectively. The total interference at the primary BS must not exceed the interference threshold $\left(I_{\mathrm{th}}\right)$. Therefore, the interference threshold constraint can be written as

$$
\sum_{i=1}^{N_{s}} P_{i} g_{i}^{(s 2 p)} \leq I_{\mathrm{th}}
$$

where $g_{i}^{(s 2 p)}$ is the power gain from the transmitter of $i$ th secondary link to the primary receiving point. We assumed that the base station has perfect knowledge about all channel state information (CSI). Some good approaches mentioned in [17] may be used to estimate these channel gains at the base station (using a signaling channel to feedback channel information to the BS). Additionally, as mentioned in [11], the channel gains can be estimated at SUs by direct reception from a primary receiver using some pilot signals or by using the band manager, which can transfer the channel information between primary and SUs. The channel gain estimator without feedback could be also utilized by the method presented in[20]. Furthermore, considering more practical situations, the impact of imperfect CSI has been investigated in Section "Impact of imperfect CSI on the throughput". The SINR requirement of each secondary link can be expressed as [17]

$$
\operatorname{SINR}_{i}=\frac{B}{R(i)} \frac{P_{i} g_{i i}^{(s 2 s)}}{\sum_{j \neq i}^{N_{s}} P_{j} g_{j i}^{(s 2 s)}+I_{i}^{p 2 s}+N_{i}} \geq Q_{i}^{*}, \quad i=1, \ldots, N_{s},
$$

where $I_{i}^{p 2 s}$ represents the interference caused by the PUs in the SUs and $N_{i}$ is the noise at the secondary link $\mathrm{i}$. Therefore, $\eta_{i}=I_{i}^{p 2 s}+N_{i}$ represents the total noise and interference due to PU transmissions at the secondary link $i$. In the above formula $B / R_{i}=G_{i}$ is the processing gain, $B$ indicates the system bandwidth, $g_{j i}^{(s 2 s)}$ denotes the link gain from transmitter $j$ to receiver $i$, and the SINR requirement of secondary link $i$ can be satisfied if SINR of $i$ th secondary link is larger than a corresponding value $Q_{i}^{*}$. Hereafter, the term "user" is utilized instead of "SU" for brevity. 


\section{RRA for elastic traffic}

In this section, RRA for elastic users (all the SUs are elastic users) is considered. Elastic flows correspond to the transfer of digital documents (e.g., web pages, emails). These flows are flexible towards rate fluctuations. The RRA is implemented in centralized fashion at the primary base station. The secondary elastic user rate allocation problem is formulated as the following optimization problem, which maximizes a certain function of secondary link transmission rate:

$$
\begin{array}{ll}
\text { Problem } & \mathcal{O}_{1} \text { : } \\
\max _{\boldsymbol{P}_{i}, \boldsymbol{R}_{i}} & f\left(R_{i}\right), \\
\text { s.t. } & \sum_{i=1}^{N_{s}} P_{i} g_{i}^{(s 2 p)} \leq I_{\mathrm{t} h}, \\
& \frac{B}{R_{i}} \frac{P_{i} g_{i i}^{(s 2 s)}}{\sum_{j \neq i} P_{j} g_{j i}^{(s 2 s)}+I_{i}^{p 2 s}+N_{i}} \geq Q_{i}^{*}, \quad i=1, \ldots, N_{s} \\
& \quad i=1, \ldots, N_{s} \\
& 0 \leq P_{i} \leq P_{i}^{\max }, \\
R_{i} \leq R_{i}^{\max }, & i=1, \ldots, N_{s} .
\end{array}
$$

The objective function $f\left(R_{i}\right)$ is designed so as to achieve efficiency while keeping a certain level of fairness. This article investigates max-min fairness and PF which have frequently been used for different resource allocation schemes (see, e.g., [17-19]). These fairness criteria are defined as follows:

$$
\begin{aligned}
& \max -\min \text { fairness } f\left(R_{i}\right)=\max \left\{\min _{i} R_{i}\right\}, \\
& \text { PF } \quad f\left(R_{i}\right)=\sum_{i=1}^{N_{s}} \ln \left(R_{i}\right)
\end{aligned}
$$

The max-min fairness criteria makes perfect fairness for all secondary links. However, the link with the worst channel condition limits the transmission rate of all secondary links. Therefore, the focus of $\max -\min$ fairness is not on maximizing the total throughput; instead, the emphasis is given to the satisfaction of fairness from the users' point of view. On the other hand, in PF, which has been received increasing attention in recent years (see, e.g., [16-21]), rate allocations are proportionally fair if they maximize sum of the logarithmic values of the rate allocations over all links. The proportional fair allocation offers a better trade off between fairness and throughput. Equations (2) and (3) are the interference threshold constraint and the SINR requirement of each secondary link, respectively, which is mentioned in Section "System model". Moreover, it is assumed that the transmission power and rate of SUs are limited by $P_{i}^{\max }$ and $R_{i}^{\max }$, respectively.

$\mathcal{O}_{1}$ is not a convex optimization problem. Using the simple transformations described in Appendix, we obtain the equivalent standard form geometric program (GP). As a result, this problem can be transformed into a convex optimization problem and solved efficiently by utilizing the convex form transformation explained in [17].

\section{RRA for streaming traffic}

This section formulated the optimal RRA based on streaming traffic requirements. Streaming traffic such as real-time applications has strict delay requirements. A queue that follows $M / G / 1$ model for each user is utilized. This model corresponds to a single server that has unlimited queue capacity and infinite calling population, while the arrival is Poisson process, meaning the statistical distribution of the inter-arrival times follow the exponential distribution and the distribution of the service time may follow any general statistical distribution. Here, the problem of resource allocation is considered with streaming traffic, which requires a maximum guaranteed average delay. We assumed that the required average maximum delay of streaming user $i$ is $\tau_{i}$. Additionally, the arrival traffic for user $i$ is modeled via a Poisson distribution with the average $\lambda_{i}$. The optimal cross-Layer RRA problem is as follows:

$$
\begin{aligned}
& \text { Problem } \mathcal{O}_{2} \text { : } \\
& \begin{array}{ll}
\max _{\boldsymbol{P}_{\boldsymbol{i}}, \boldsymbol{R}_{\boldsymbol{i}}} & f\left(R_{i}\right), \\
\text { s.t. } & \sum_{i=1}^{N_{s}} P_{i} g_{i}^{(s 2 p)} \leq I_{\mathrm{t} h}, \\
& G_{i} \frac{P_{i} g_{i i}^{(s 2 s)}}{N_{s}} P_{j} g_{j i}^{(s 2 s)}+I_{i}^{p 2 s}+N_{i}^{*}, \quad i=1, \ldots, N_{s} \\
& \sum_{j \neq i} \\
& 0 \leq P_{i} \leq P_{i}^{\max }, \\
& R_{i} \leq R_{i}^{\max }, \\
& \mathbb{E}\left(W_{i}\right) \leq \tau_{i} \\
& i=1, \ldots, N_{s}
\end{array}
\end{aligned}
$$

Delay constraint for streaming users is shown in (13), in which $\mathbb{E}\left(W_{i}\right)$ is the average waiting time of user $i$ in the queue plus the service time. In the optimization problem, it is assumed that users have enough traffic waited in the queue and ready to be transmitted (i.e., full buffer assumption).

In order to solve the cross-layer resource allocation problem, $\mathcal{O}_{2}$, the delay constraint (13) is converted into a 
constraint in terms of physical-layer parameters. To transform the constraint in (13), according to [22] an equation is obtained which models the relationship between the scheduled data rate of the user $i$ and its traffic characteristic $\left(\tau_{i}, \lambda_{i}\right)$. As mentioned in [23] for $M / G / 1$ model

$$
\mathbb{E}\left(X_{i}\right)+\frac{\lambda_{i} \mathbb{E}\left(X_{i}^{2}\right)}{2\left(1-\lambda_{i} \mathbb{E}\left(X_{i}\right)\right)} \leq \tau_{i},
$$

where $X_{i}$ is the service time of user $i$. By mathematical derivations based on (14) which is provided in [22], the following constraint is obtained:

$$
R_{i} \geq \psi\left(\mathbb{E}(z), \tau_{i}, \lambda_{i}\right)
$$

where

$$
\psi\left(\mathbb{E}(z), \tau_{i}, \lambda_{i}\right)=\frac{2 \lambda_{i} \mathbb{E}(z)}{\left(2+2 \lambda_{i} \tau_{i}\right)-\sqrt{\left(2+2 \lambda_{i} \tau_{i}\right)^{2}-8 \lambda_{i} \tau_{i}}},
$$

and $\mathbb{E}(z)$ is the average packet size. As a result, the optimization problem $\mathcal{O}_{2}$ is transformed into the following:

$$
\begin{aligned}
& \text { Problem } \mathcal{O}_{3}: \\
& \max _{\boldsymbol{P}_{i}, \boldsymbol{R}_{i}} f\left(R_{i}\right), \\
& \text { s.t. } \quad \sum_{i=1}^{N_{s}} P_{i} g_{i}^{(s 2 p)} \leq I_{\mathrm{t} h}, \\
& \quad G_{i} \frac{P_{i} g_{i i}^{(s 2 s)}}{N_{s}} P_{j} g_{j i}^{(s 2 s)}+I_{i}^{p 2 s}+N_{i} \\
& \quad \sum_{j \neq i}^{*}, \quad i=1, \ldots, N_{s} \\
& \\
& 0 \leq P_{i} \leq P_{i}^{\max }, \\
& R_{i} \leq R_{i}^{\max }, \\
& R_{i} \geq \psi\left(\mathbb{E}(z), \tau_{i}, \lambda_{i}\right),
\end{aligned}
$$

where constraint (13) in $\mathcal{O}_{2}$ is substituted by (22) in this optimization problem. The original problem formulation presented in $\mathcal{O}_{2}$ is a cross-layer optimization problem, which considers different delay requirements. In other words in this formulation, we translated MAClayer parameters into physical-layer parameters. Similar to section "RRA for elastic traffic" we adopt GP to obtain the solution of the optimization problem in $\mathcal{O}_{3}$.

\section{RRA for heterogeneous traffic}

In this section, a mixture of two traffic types including streaming and elastic is considered. The total number of SUs, $N_{s}$, is divided into two groups: (1) SUs with streaming traffic, where $k_{i}^{s}$ is the $i$ th secondary streaming user and $u^{s}$ is the number of secondary streaming users; and (2) SUs with elastic traffic, where $k_{i}^{e}$ is the $i$ th secondary elastic user and $u^{e}$ is the number of secondary elastic users so that $u^{e}+u^{s}=N_{s}$.

Corresponding to each user, a queue that follows $M / G / 1$ model is considered. Here the problem of resource allocation is considered with streaming traffic, which requires a maximum guaranteed average delay, and elastic traffic with flexible rate of requirements. The main objective is to maximize $f\left(R_{k_{i}^{e}}\right)$ while the average delay constraint and QoS constraint for streaming traffic and, the maximum transmission power constraint and interference threshold constraint are satisfied.

It is assumed that the required average maximum delay of streaming user $k_{i}^{s}$ is $\tau_{k_{i}^{s}}$. Moreover, the arrival traffic for user $k_{i}^{s}$ is modeled via a Poisson distribution with the average $\lambda_{k_{i}^{s}}$. The optimal cross-Layer RRA problem is as follows:

\section{Problem $\mathcal{O}_{4}:$}

$$
\begin{aligned}
& \max _{\boldsymbol{P}_{i}, \boldsymbol{R}_{i}} f\left(R_{k_{i}^{e}}\right), \\
& \text { s.t. } \quad R_{k_{i}^{s}} \geq R_{m} \\
& \sum_{i=1}^{u^{e}} P_{k_{i}^{e}} g_{k_{i}^{e}}^{(s 2 p)}+\sum_{i=1}^{u^{s}} P_{k_{i}^{s}} g_{k_{i}^{s}}^{(s 2 p)} \leq I_{\mathrm{t} h} \\
& \frac{B P_{k_{i}^{s}} g_{k_{i i}^{s}}^{(s 2 s)}}{R_{k_{i}^{s}}\left(\sum_{j \neq i}^{u^{s}} P_{k_{j}^{s}} g_{k_{j i}^{s}}^{(s 2 s)}+\sum_{j}^{u^{e}} P_{k_{j}^{e}} g_{k_{j i}^{e}}^{(s 2 s)}+I_{i}^{p 2 s}+N_{i}\right)} \geq Q_{i}^{*}, \\
& i=1, \ldots, u^{s} \\
& \frac{B P_{k_{i}} g_{k_{i i}^{e}}^{(22 s)}}{R_{k_{i}^{e}}\left(\sum_{j \neq i}^{u^{e}} P_{k_{j}^{e}} g_{k_{i j}^{e}}^{(s 2 s)}+\sum_{j}^{u^{s}} P_{k_{j}} g_{k_{j i}^{s}}^{(s 2 s)}+I_{i}^{p 2 s}+N_{i}\right)} \geq Q_{i}^{*}, \\
& i=1, \ldots, u^{e} \\
& 0 \leq P_{k} \leq P^{\max }, \quad k \in\left\{k_{1}^{s} \ldots, k_{u^{s}}^{s}, k_{1}^{e}, \ldots, k_{u^{e}}^{e}\right\} \\
& R_{k} \leq R^{\max }, \quad k \in\left\{k_{1}^{s}, \ldots, k_{u^{s}}^{s}, k_{1}^{e}, \ldots, k_{u^{e}}^{e}\right\}
\end{aligned}
$$

where delay constraint for streaming users as previously described in section "RRA for streaming traffic" can be written as (24), in which $R_{k_{i}^{s}}$ is the transmission rate of user $k_{i}^{s}$ and $R_{m}=\psi\left(\mathbb{E}(z), \tau_{k_{i}^{s}}, \lambda_{k_{i}^{s}}\right)$ is the minimum rate requirement for each streaming users; (25) represents the interference threshold constraint for both elastic and streaming users as defined in previous sections, (26) and (27) states SINR constraint for streaming and elastic traffic. Equations (28) and (29) are the total transmission power and rate constraints. Similar to Section "RRA for streaming traffic", we adopt GP to obtain the solution of the optimization problem in $\mathrm{O}_{4}$. 


\section{Simulation results}

A single-cell CDMA system with $5 \mathrm{MHz}$ channel bandwidth is considered. The maximum transmission power on secondary links is $0.1 \mathrm{~W}$. The minimum transmission rate requirement of the streaming users is set to $128 \mathrm{kbps}$. The maximum transmission rate is $R^{\max }=$ $B / P G m i n$, where $P G m i n$ is the minimum processing gain. This equation shows that by increasing the minimum processing gain, the $R^{\max }$ decreases.

The channel gains are modeled as $K_{0} 10^{\gamma / 10}\left(d^{-4}\right)$ where $d$ is the distance between the transmitter and the receiver, $\gamma$ is random Gaussian variables with zero mean and the standard deviation equal $6 \mathrm{~dB}, K_{0}=10^{3}$ which captures system and transmission effects such as antenna gain, carrier frequency, etc. The total noise and interference at the receiving nodes of all secondary links is chosen to be $\eta_{i}=\eta=10^{-10} \mathrm{~W}$.

PUs communicate with its base station in the uplink direction. Transmitting nodes corresponding to the secondary links are randomly located in a coverage area with the BS of primary network located at the center. The size of coverage area is $4 \mathrm{~km}^{2}$. Furthermore, the receiving node of each secondary link is generated randomly in a $1 \mathrm{~km} \times 1 \mathrm{~km}$ rectangle with its transmitting node being at the center.

\section{Impact of different traffics on throughput}

In Figures 2 and 3, the total throughput of SUs for PF and max-min fairness versus the minimum processing gain under different SINR constraints are illustrated. Two cases are considered. First, it is assumed that all the SUs are

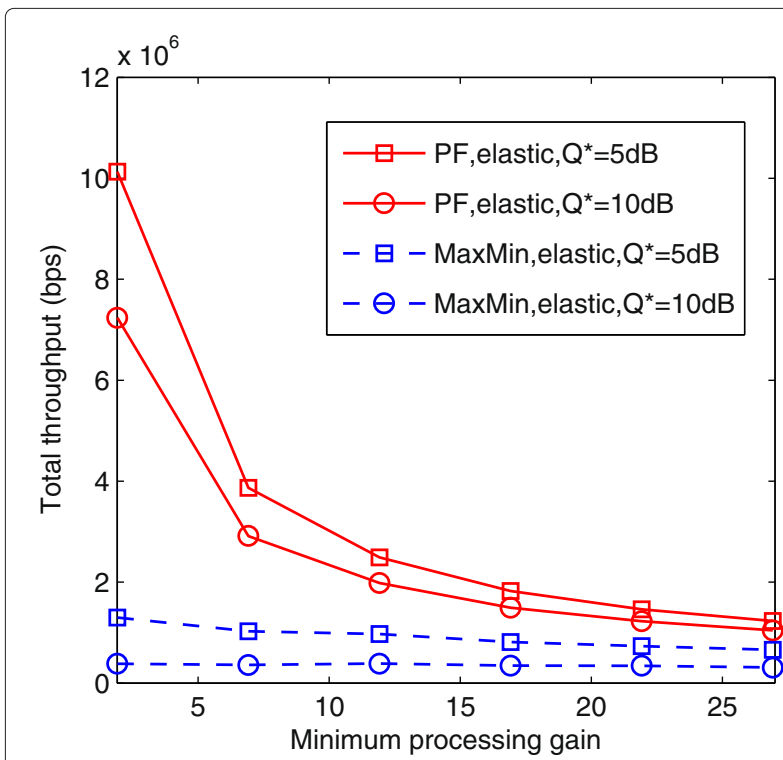

Figure 2 Total throughput of elastic users versus the minimum processing gain for PF and max-min fairness with $I_{t h}=20 \eta$. All SUs are elastic users.

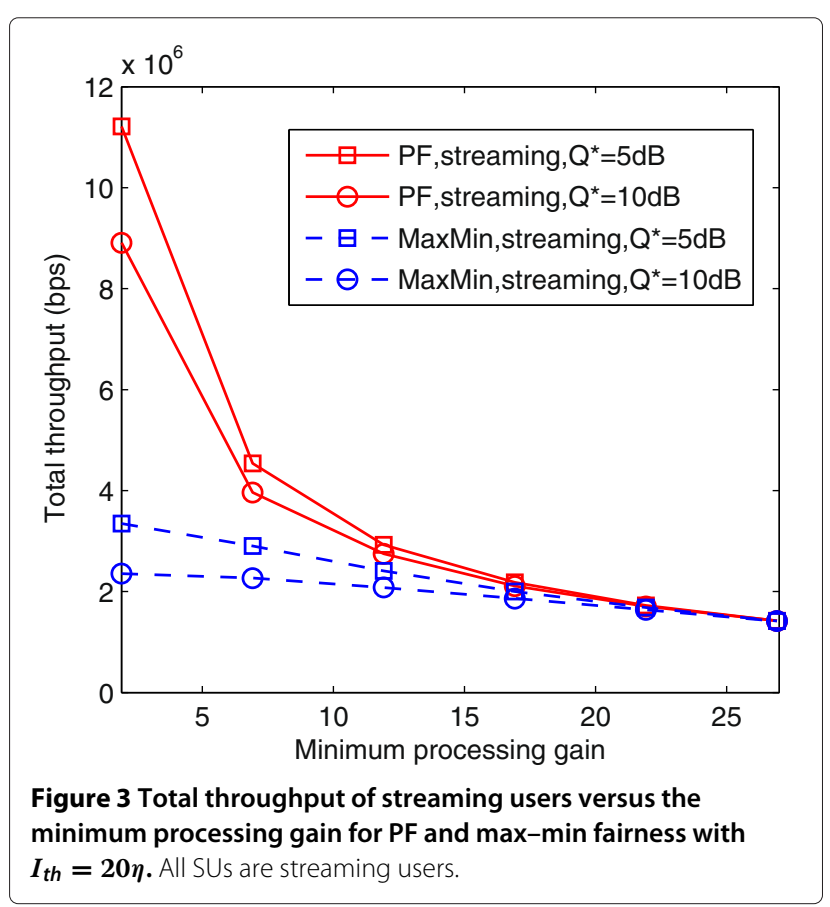

elastic users. The total throughput of elastic users are evaluated in Figure 2. Then it is considered that all the SUs are streaming users. Figure 3 depicts total throughput of streaming users versus the minimum processing gain. The total number of SUs set to 10. It is seen that joint rate and power allocation with PF achieve higher throughput than of the max-min fairness criteria for both streaming and elastic cases. This is due to the fact that in maxmin fairness the link with the worst channel condition limits the transmission rate of all secondary links. Furthermore, it can be observed that the more stringent is the SINR constraints, the lower is the throughput that can be achieved. We compare two target SINRs (the minimum SINR requirements) which were selected as $Q^{*}=5 \mathrm{~dB}$ and $Q^{*}=10 \mathrm{~dB}$. As it is observed, by increasing the SINR constraint, the rate of users decreases; hence, the total throughput of users decreases. For a given minimum processing gain $(P G \min =2)$, while target SINR increases from 5 to $10 \mathrm{~dB}$ the total throughput of elastic users for PF decrease from 10.13 to $7.2 \mathrm{Mbps}$ and the total throughput of streaming users decrease from 11.21 to $8.91 \mathrm{Mbps}$. Furthermore, for max-min fairness the total throughput of elastic users decrease from 1.3 to $0.38 \mathrm{Mbps}$ and the total throughput of streaming users decrease from 3.3 to 2.35 Mbps.

In addition, throughput of the secondary network decreases when minimum processing gain is increased. This is due to the fact that maximum data rates decrease when the minimum processing gain increases. If the PGmin varies from 2 to 27, the total throughput of elastic users in max min decrease from 1.3 to $0.66 \mathrm{Mbps}$ and the 
total throughput of streaming users in max-min decrease from 3.3 to $1.42 \mathrm{Mbps}$. Moreover, the performance gap of total throughput become smaller. This results from the fact that, when the minimum processing gain increases, the maximum rate decreases which essentially reduces the feasible region. Hence, the gaps among different throughput curves become smaller.

Comparing the results of Figure 2 to those of Figure 3, it can be seen that streaming traffic achieve higher throughput than of the elastic traffic for both PF and max-min fairness. This results from the fact that, for streaming applications, stringent transmission rate guarantees are necessary to ensure real-time communication.

\section{Impact of different traffics on fairness}

To examine the fairness attribute of the resource allocation solutions for secondary links, the fairness index versus the minimum processing gains is plotted for both elastic and streaming users in Figures 4 and 5. Jain quantitative measure is utilized which is define as follows:

$$
F I_{\mathrm{Jain}}=\frac{\left(\sum_{i=1}^{N_{s}} R_{i}\right)^{2}}{N_{s} \sum_{i=1}^{N_{s}} R_{i}^{2}} .
$$

This index is applicable to any resource allocation problem and has been used variously in the literature (see, e.g., $[17,19])$. It is independent of the amount of available resources. In other words, it is dimensionless and independent of scale and is also a continuous parameter. It is bounded between 0 (for the worst case) and 1 (for the

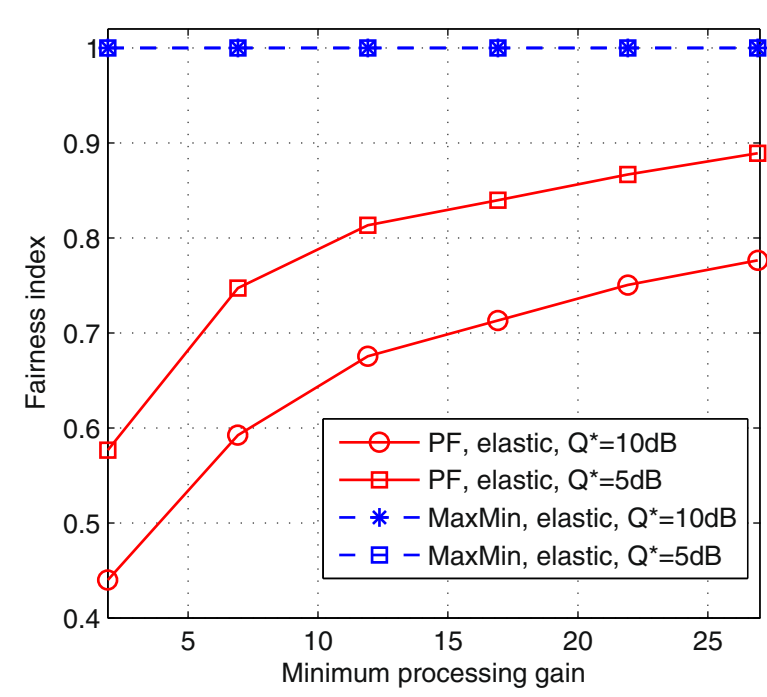

Figure 4 Fairness index of SUs versus the minimum processing gain with $I_{t h}=20 \eta$. All SUs are elastic user.

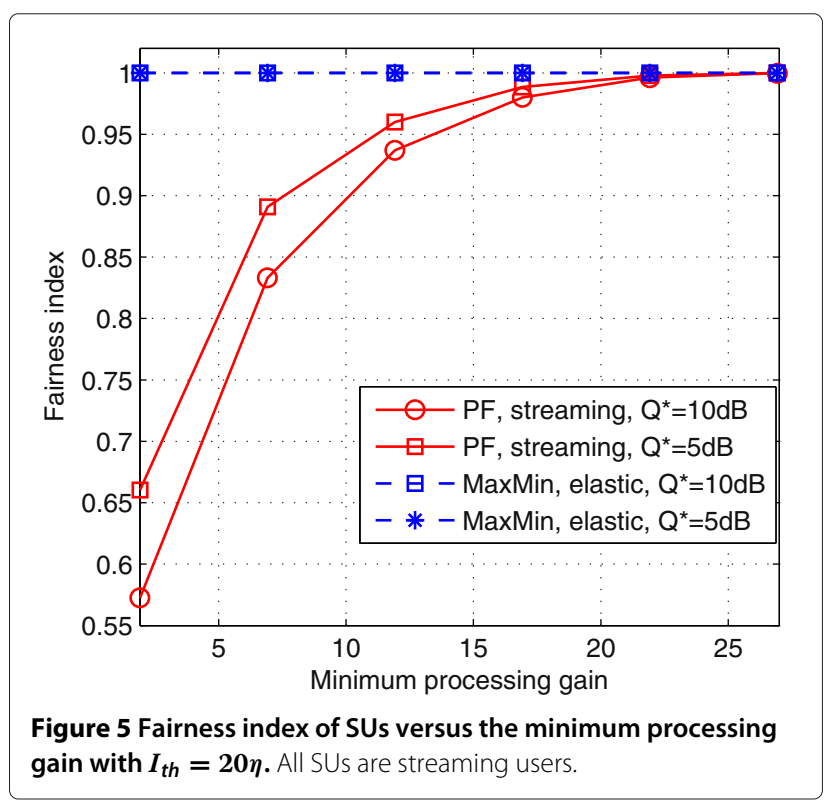

best case). If all users get the same amount, then the fairness index is equal to one (when all users receive the same resource allocation), and the system is $100 \%$ fair. Maxmin fairness is an instance of the systems with the fairness index of $100 \%$.

It is apparent from Figures 4 and 5 that resource allocation solutions are fairer for the streaming case in comparison to elastic users. This is due to the fact that all streaming users must satisfy the rate constraint. In other words, all the streaming users achieve their minimum rates. As a result, we can note that the addition of the rate constraints (adding a minimum rate constraint for streaming case) has improved the fairness in the system.

Furthermore, fairness improves when the minimum processing gain increases (i.e., due to smaller feasible rate region). Moreover, as is observed from Figure 4 the more stringent is the SINR constraints, the lower is the fairness that can be achieved. However, fairness curves of streaming users in comparison to curves of elastic users get closer to each other as the minimum processing gain increases because of smaller feasible rate region for the case of streaming users.

\section{Impact of different traffics on sum power}

The sum power of SUs versus the minimum processing gain is demonstrated in Figure 6. As it is seen, the sum power of SUs in elastic traffic is less than that of the streaming traffic due to lower rate and power allocated to the elastic users. In addition, the sum power of PF is higher than the sum power of the max-min fairness due to higher rate and power allocated to users in PF. 


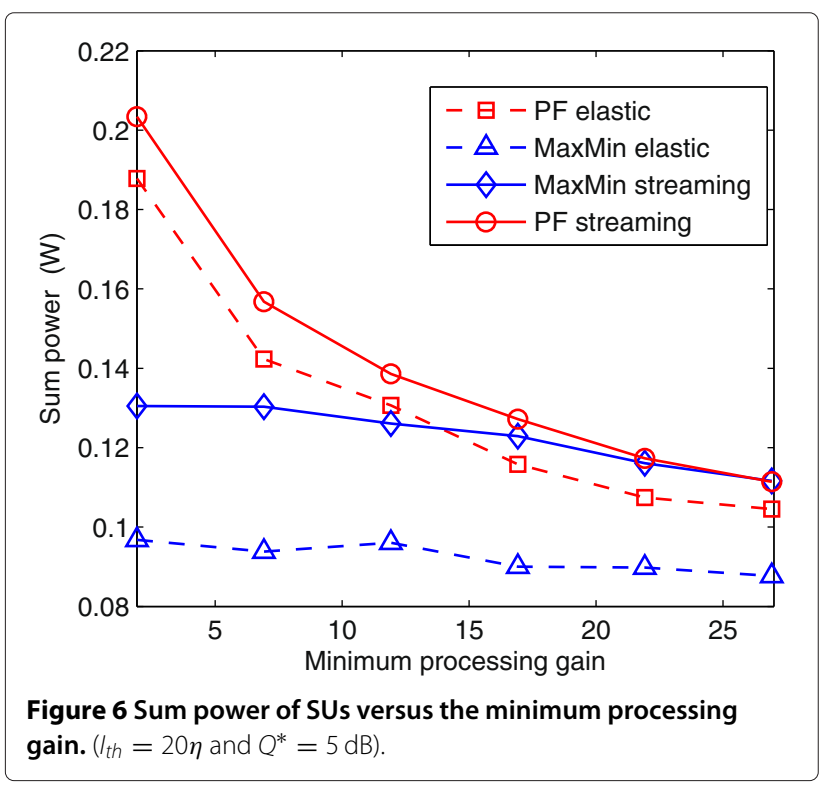

\section{Effect of heterogeneous traffic}

In this section, we consider a mixture of elastic and streaming traffic. To investigate the effect of streaming traffic on the total throughput of elastic users, we consider different numbers of streaming traffic $\left(U_{s}=2,4,6\right)$. The results are demonstrated in Figure 7 which shows the total throughput of elastic users versus interference threshold. The solid and dashed lines represent PF and $\max$-min fairness techniques, respectively. In this figure,

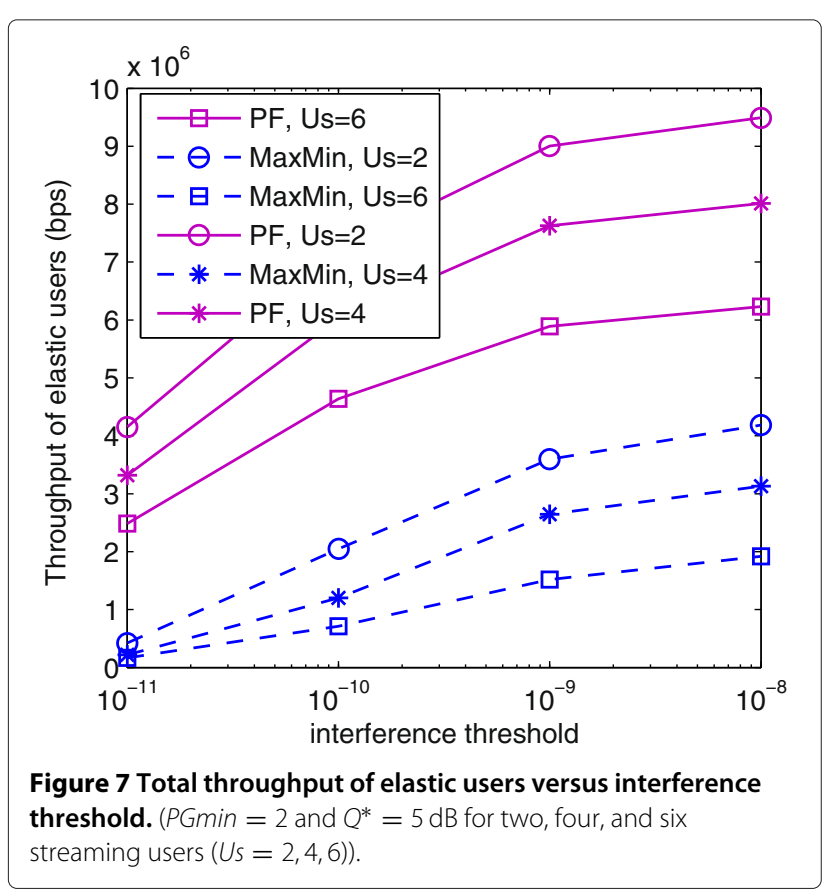

the minimum processing gain has been set to 2 and the rate requirement of streaming users $=128 \mathrm{kbps}$. As can be seen, increasing the number of streaming traffic results in lower throughput of elastic users for both PF and maxmin fairness due to the fact that less elastic users are competing with more streaming users for the network resources. The figure also reveals that as the interference threshold increases, the allocated power for elastic users, hence, their achievable rate and, consequently, throughput of elastic users also increase.

We also performed simulations for streaming users. Figure 6 illustrates that the rate of streaming users is almost constant for different interference threshold levels which is very important for keeping the QoS of streaming users consistently. It can be also observed from these plots that as the number of streaming users increases, the total throughput also increases.

We further examine the effect of different SINR constraint on the total throughput of elastic users (Figure 8). Plots for the total throughput of elastic users versus the interference threshold under various SINR constraints $(Q *=5,10,15 \mathrm{~dB})$ with $U_{s}=4$ for $\mathrm{PF}$ and max-min fairness are provided in Figure 9. We observe that by increasing the SINR constraint, according to (27) the rate of elastic users decreases, thereby, the total throughput of elastic users decreases for both PF and max-min fairness; however, gap in the throughput graphs, decreases for lower values of interference threshold due to smaller feasible rate region. For instant, by increasing the SINR constraint form $Q *=5$ to $Q *=15 \mathrm{~dB}$, for a given interference threshold $\left(10^{-8}\right)$ the throughput

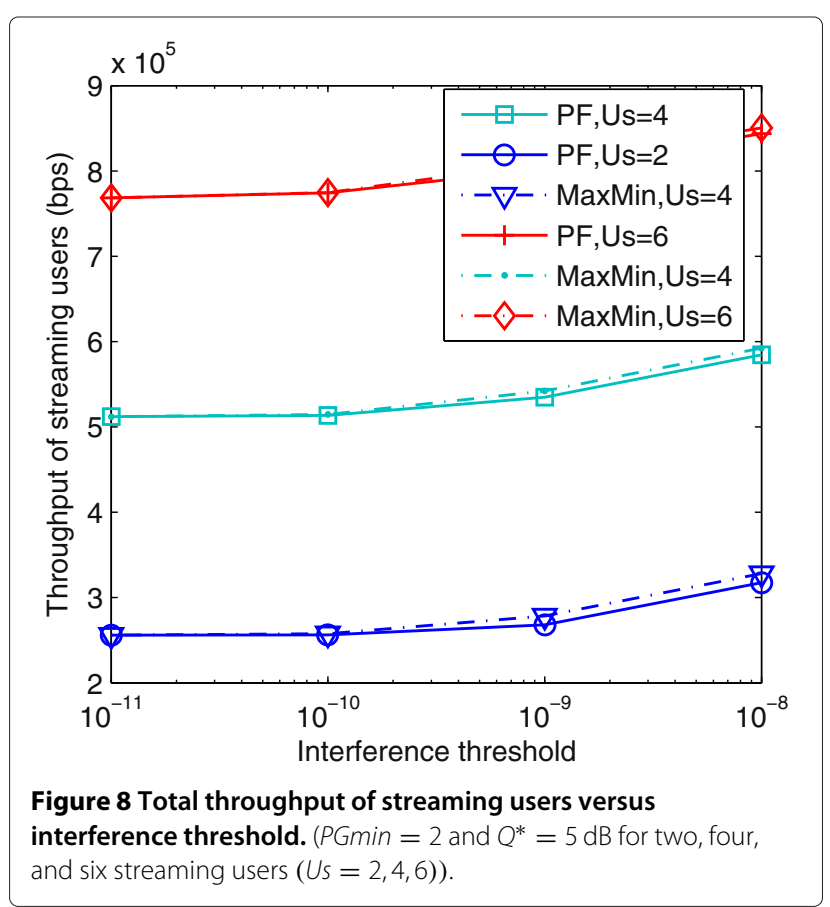




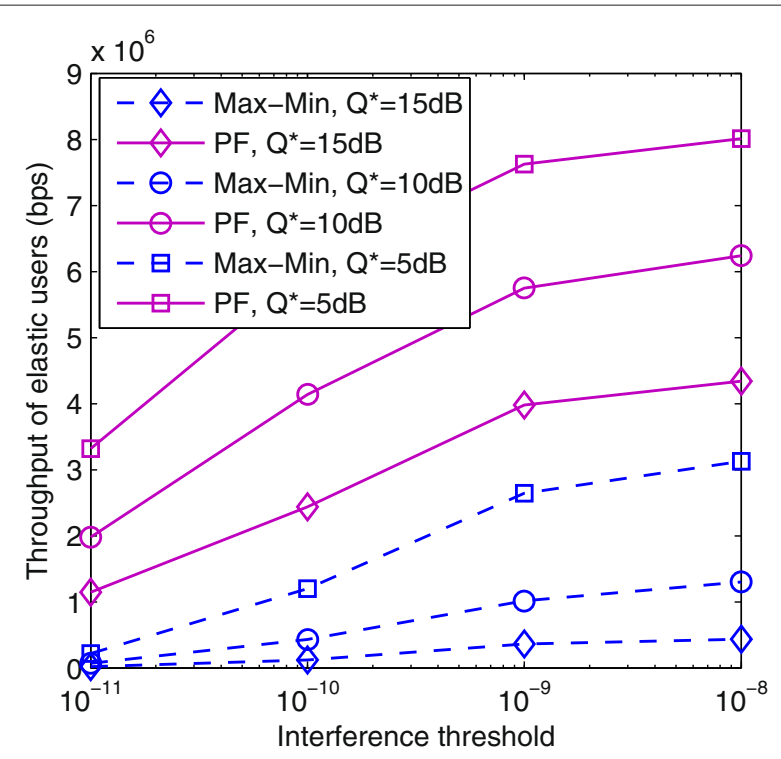

Figure 9 Total throughput of elastic users versus interference threshold for four streaming users. ( $P G$ min $=2$ and different SINR constraint $\left.\left(Q^{*}=5,10,15 \mathrm{~dB}\right)\right)$.

degradation is about 4 and $2.5 \mathrm{Mbps}$ for PF and maxmin fairness, respectively. In addition, for a given interference threshold $\left(10^{-11}\right)$ the throughput degradation is about 2 Mbps and $150 \mathrm{kbps}$ for PF and max-min fairness, respectively.

Figure 10 includes the plots for the total throughput of elastic users versus the interference threshold under various $P$ Gmins, with $U_{s}=4$ for PF and max

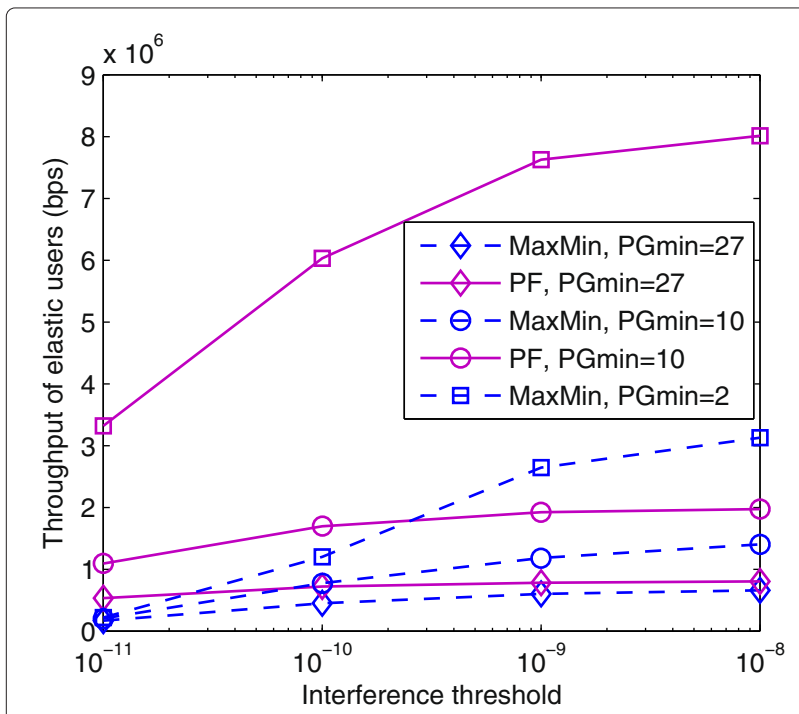

Figure 10 Total throughput of elastic users versus interference threshold for four streaming users. $\left(Q^{*}=5 \mathrm{~dB}\right.$ and under different minimum process gain $(P G \min =2,10,27))$. min fairness. Throughput curves indicate that throughput of elastic users decreases when the minimum processing gain increases. This results from the fact that as the minimum processing gain increases, the maximum rate decrease, hence, total throughput of elastic users decreases. However, the rate of throughput growth is not the same for all amount of interference threshold owing to the fact that as the interference threshold further increases, the elastic users make more interference to PUs. Then the SU increases its transmission power whereas this is finally limited by its maximum transmission power. Consequently, further increase of the interference threshold does not help the elastic users to transmit higher power. Hence, the higher level of the interference threshold (more than $10^{-8}$ ) have negligible effects on total throughput of elastic users.

\section{Impact of imperfect CSI on the throughput}

Due to the technical reasons such as estimation errors and wireless channel delay, perfect CSI is not available. Therefore, it is assumed that imperfect CSI is available at the secondary station. Then the radio resources are allocated based on an imperfect estimation of the channel gain by $\hat{g}^{(s 2 s)}$ and $\hat{g}^{(s 2 p)}$, so that [24]

$$
\begin{gathered}
g^{(s 2 s)}=\hat{g}^{(s 2 s)}+\Delta_{s 2 s} \\
g^{(s 2 p)}=\hat{g}^{(s 2 p)}+\Delta_{s 2 p} .
\end{gathered}
$$

where $\Delta_{s 2 s}$ and $\Delta_{s 2 p}$ stands for the estimation error, where these are usually modeled by a zero mean complex Gaussian random variable with variance $\sigma_{s 2 s}^{2}$ and $\sigma_{s 2 p}^{2}$, respectively. We examine the effect of imperfect CSI on the throughput of the elastic and streaming users. Plots for the throughput of the elastic and streaming users for max-min fairness versus the minimum processing gain are provided in Figure 11. As it is observed due to imperfect CSI assumption, the throughput of the both elastic users and streaming users decrease. It is also seen, due to smaller feasibility region, increasing the minimum processing gain, results in decreasing the throughput. Figure 12 shows that the same results can be achieved using PF. This figure also reveals that imperfect CSI degrades the total throughput of the users.

This figure also demonstrates that the total throughput of the SUs in PF is higher than the total throughput of the SUs in the max-min fairness. This results from the fact that in max-min fairness criterion the link with the worst channel condition limits the transmission rate of all secondary links. This is due to the fact that max-min fairness gives an absolute priority to flows with small bit rates. In wireless networks, this results in an inefficient allocation 


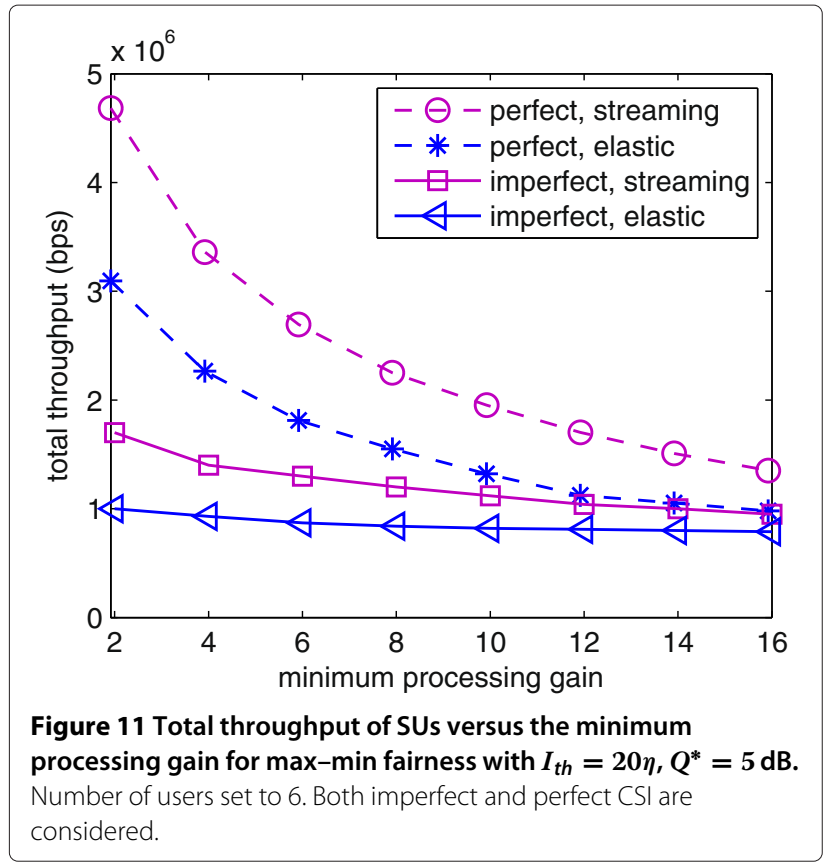

where flows that experience bad radio conditions consume most radio resources.

\section{Conclusion}

Rate and power allocation problem for SUs with QoS requirements and interference threshold constraint in CDMD-based CR system has been formulated as an optimization problem. Three scenarios have been considered. The first scenario corresponds to elastic traffic

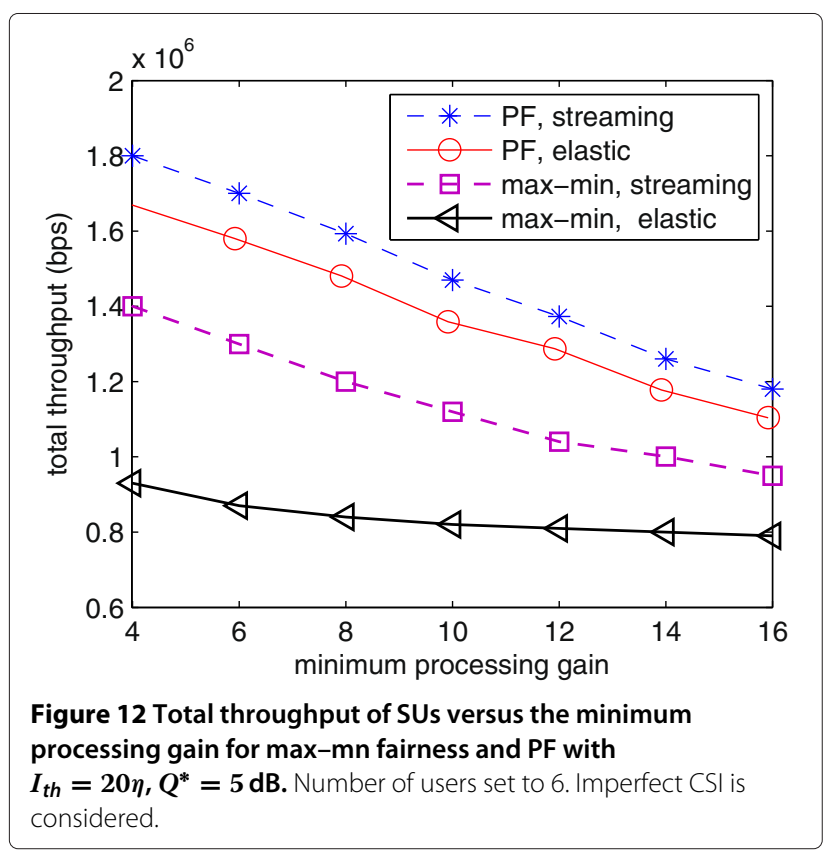

in which all the SUs are elastic users. The GP has been proposed for solving the problem. In the second, it is assumed that all the SUs are streaming users. To solve this optimization problem, the delay constraints are translated into physical-layer parameters so that cross-layer optimization problem converted into a physical-layer optimization problem. Considering the PF and max-min fairness, it has been observed that for both fairness criterion the streaming users achieving higher throughput and fairness, compared with the elastic users due to the fact that for streaming users, stringent transmission rate guarantees are necessary to ensure real-time communication.

Heterogeneous traffic (mixture of elastic and streaming traffic) has been considered with regard to the future requirements of next-generation wireless networks in the third scenario. Simulations have been used for demonstrating the effect of streaming traffic, interference threshold, imperfect CSI, minimum processing gain, and SINR constraint on the total throughput of elastic users. Moreover, it is shown that for all the three scenarios PF outperforms max-min fairness from throughput point of view.

\section{Appendix}

The objective function of PF is to maximize $\sum_{i=1}^{N_{s}} \ln \left(R_{i}\right)$ which is equivalent to maximize $\ln \left(\prod_{i=1}^{N_{s}}\left(R_{i}\right)\right)$ and then can be rewritten as minimize $\frac{1}{\prod_{i=1}^{N_{S}}\left(R_{i}\right)}$. Therefore, the optimization problem can be express as following geometric problem:

$$
\begin{aligned}
& \min _{\boldsymbol{P}_{i}, \boldsymbol{R}_{i}} \prod_{i=1}^{N_{s}}\left(R_{i}^{-1}\right), \\
& \text { s.t. } \quad \sum_{i=1}^{N_{s}} \frac{P_{i} g_{i}^{(s 2 p)}}{I_{t h}} \leq 1, \\
& R_{i} P_{i}^{-1} \frac{Q_{i}^{*}}{B g_{j i}^{(s 2 s)}} \sum_{j \neq i}^{N_{s}} P_{j} g_{j i}^{(s 2 s)}+R_{i} P_{i}^{-1} \frac{Q_{i}^{*} I_{i}^{p 2 s}}{B g_{j i}^{(s 2 s)}} \\
& \quad+R_{i} P_{i}^{-1} \frac{Q_{i}^{*} N_{i}}{B g_{j i}^{(s 2 s)}} \leq 1, \quad i=1, \ldots, N_{s} \\
& \left(P_{i}^{\max }\right)^{-1} P_{i} \leq 1, \quad i=1, \ldots, N_{s} \\
& \left(R_{i}^{\max }\right)^{-1} R_{i} \leq 1, \quad i=1, \ldots, N_{s} .
\end{aligned}
$$

The variables are defined as $y_{i}=\log R_{i}$ so $R_{i}=e^{y_{i}}$ and $z_{i}=\log p_{i}$ so $p_{i}=e^{z_{i}}$. After the change of variables, a polynomial becomes a sum of exponentials of affine 
functions. The above GP can be rewritten in terms of new variables and by considering the logarithm as:

$$
\begin{array}{ll}
\min _{\boldsymbol{p}_{\boldsymbol{i}}, \boldsymbol{R}_{\boldsymbol{i}}} & \log \left(\sum_{i=1}^{N_{s}} e^{-y_{i}}\right) \\
\text { s.t. } \quad & \log \left(\sum_{i=1}^{N_{s}} \frac{g_{i}^{(s 2 p)}}{I_{t h}} e^{z_{i}}\right) \leq 0, \\
& \log \left(\frac{Q_{i}^{*}}{B g_{i i}^{(s 2 s)}} \sum_{j \neq i}^{N_{s}} e^{\left(y_{i}+z_{j}-z_{i}\right)} g_{j i}^{(s 2 s)}+\frac{Q_{i}^{*} I_{i}^{p 2 s}}{B g_{j i}^{(s 2 s)}} e^{\left(y_{i}-z_{i}\right)}\right. \\
& \left.+\frac{N_{i} Q_{i}^{*}}{B g_{i i}^{(s 2 s)}} e^{\left(y_{i}-z_{i}\right)}\right) \leq 0, \quad i=1, \ldots, N_{s}, \\
& \log \left(\left(P_{i}^{\max }\right)^{-1} e^{z_{i}}\right) \leq 0, i=1, \ldots, N_{s}, \\
& \log \left(\left(R_{i}^{\max }\right)^{-1} e^{y_{i}}\right) \leq 0, i=1, \ldots, N_{s} .
\end{array}
$$

Since log-exp and log-sum-exp functions are convex, the above optimization problem is a convex optimization problem [25]. Therefore, the global optimal solution can be achieved by interior point methods [17], which solve the problem by applying Newton's method to a sequence of equality constrained problems.

\section{Competing interests}

The authors declare that they have no competing interests.

\section{Author details}

${ }^{1}$ Department of Electrical Engineering, Science and Research Branch, Islamic Azad University, Tehran, Iran. ${ }^{2}$ Electrical and Computer Engineering

Department, Tarbiat Modares University, Tehran, Iran.

Received: 11 January 2012 Accepted: 25 July 2012

Published: 17 August 2012

\section{References}

1. S Haykin, Cognitive radio: brain-empowered wireless communications. IEEE J. Sel. Areas Commun. 23(2), 201-220 (2005)

2. S Aslam, K Lee, Fair, efficient, and power-optimized spectrum sharing scheme for cognitive radio networks. EURASIP J. Wirel. Commun. Netw. (2011). http://jwcn.eurasipjournals.com/content/2011/1/201

3. ZYZ Peipei, C Qinyu, W Ye, Performance analysis of spectrum sharing mechanisms in cognitive radio networks. EURASIP J. Wirel. Commun. Netw ((2011). http://jwcn.eurasipjournals.com/content/2011/1/129)

4. M Dashouk, M Alanyali, A utility-based approach for secondary spectrum sharing. EURASIP J. Wirel. Commun. Netw. (2011). http://jwen. eurasipjournals.com/content/2011/1/32

5. A Molisch, Wireless Communications. (Wiley-IEEE, USA, 2011)

6. X Zhang, H Su, Opportunistic spectrum sharing schemes for CDMA-based uplink MAC in cognitive radio networks. IEEE J. Sel. Areas Commun. 29(4), 716-730 (2011)

7. M Li, SN Batalama, DA Pados, T Melodia, MJ Medley, JD Matyjas, Cognitive code-division links with blind primary-system identification. IEEE Trans. Wirel. Commun. 10(11), 3743-3753 (2011)

8. V Chakravarthy, X Li, Z Wu, MA Temple, F Garber, R Kannan, A Vasilakos, Novel overlay/underlay cognitive radio waveforms using SD-SMSE framework to enhance spectrum efficiency_Part I: theoretical framework and analysis in AWGN channel. IEEE Trans. Wirel. Commun. 57(12), 3794-3804 (2009)

9. A Attar, MR Nakhai, AH Aghvami, Cognitive radio transmission based on direct sequence MC-CDMA. IEEE Trans. Wirel. Commun. 7(4), 1157-1162 (2008)
10. X Kang, H Garg, YC Liang, R Zhang, Optimal power allocation for OFDM-based cognitive radio with new primary transmission protection criteria. IEEE Trans. Wirel. Commun. 9(6), 2066-2075 (2010)

11. J Lee, H Wang, J Andrews, D Hong, Outage probability of cognitive relay networks with interference constraints. IEEE Trans. Wirel. Commun. 10(2), 390-395 (2011)

12. B Yang, G Feng, Y Shen, C Long, X Guan, Channel-aware access for cognitive radio networks. IEEE Trans. Veh. Technol. 58(7), 3726-3737 (2009)

13. J Lotze, S Fahmy, J Noguera, L Doyle, A model-based approach to cognitive radio design. IEEE J. Sel. Areas Commun. 29(2), 455-468 (2011)

14. B Wang, D Zhao, Performance analysis in CDMA-based cognitive wireless networks with spectrum underlay. in Proceedings of the IEEE Global Telecommun. Conf. (GLOBECOM) (New Orleans, 2008), pp. 1-6

15. AT Hoang, YC Liang, A two-phase channel and power allocation scheme for cognitive radio networks. in Proceedings of the IEEE 17th International Personal, Indoor and Mobile Radio Communications Symposium (Helsinki, 2006), pp. 1-5

16. Y Xing, CN Mathur, MA Haleem, R Chandramouli, K Subbalakshmi, Dynamic spectrum access with QoS and interference temperature constraints. IEEE Trans. Mob. Comput. 6(4), 423-433 (2007)

17. L Le, E Hossain, Resource allocation for spectrum underlay in cognitive radio networks. IEEE Trans. Wirel. Commun. 7(12), 5306-5315 (2008)

18. DI Kim, L Le, E Hossain, Joint rate and power allocation for cognitive radios in dynamic spectrum access environment. IEEE Trans. Wirel. Commun. 7(12), 5517-5527 (2008)

19. B Wang, D Zhao, Scheduling for long term proportional fairness in a cognitive wireless network with spectrum underlay. IEEE Trans. Wirel. Commun. 9(3), 1150-1158 (2010)

20. K Kamdi, W Zhang, KB Letaief, Power control in cognitive radio systems based on spectrum sensing side information. in Proceedings of the IEEE International Conference on Communications (Glasgow, 2007), pp. 5161-5165

21. $Y$ Zhang, $C$ Leung, A distributed algorithm for resource allocation in OFDM cognitive radio systems. IEEE Trans. Veh. Technol. 60(2), 546-554 (2011)

22. N Mokari, MR Javan, K Navaie, Cross-layer resource allocation in OFDMA systems for heterogeneous traffic with imperfect CSI. IEEE Trans. Veh. Technol. 59(2), 1011-1017 (2010)

23. L Kleinrock, Queueing Systems. Vol. I, Chap. 10 (John Wiley and Sons, New York, 1975), p. 190

24. N Mokari, K Navaie, MG Khoshkholgh, Downlink radio resource allocation in OFDMA spectrum sharing environment with partial channel state information. IEEE Trans. Wirel. Commun. 10(10), 3482-3495 (2011)

25. S Boyd, L Vandenberge, Convex Optimization. (Cambridge University Press, Cambridge, 2004)

doi:10.1186/1687-1499-2012-262

Cite this article as: Dashti and Azmi: Joint power and rate allocation in CDMA-based underlay cognitive radio networks for a mixture of streaming and elastic traffic. EURASIP Journal on Wireless Communications and Networking 2012 2012:262 